Laser Chem., 1999, Vol. 19, pp. 19-34

Reprints available directly from the publisher Photocopying permitted by license only
(C) 1999 OPA (Overseas Publishers Association) N.V.

Published by license under

the Harwood Academic Publishers imprint, part of The Gordon and Breach Publishing Group.

Printed in India.

\title{
MYOGLOBIN DYNAMICS MEASURED WITH VIBRATIONAL ECHO EXPERIMENTS
}

\author{
K. D. RECTOR and M. D. FAYER* \\ Department of Chemistry, Stanford University, Stanford, CA 94305
}

(Received 18 August 1997)

\begin{abstract}
Ps infrared vibrational echo experiments on myoglobin and myoglobin mutants are presented. The vibrational dephasing experiments examine the influence of protein dynamics on the $\mathrm{CO}$ ligand, at the active site of myoglobin, from low temperature to physiologically relevant temperatures. The vibrational echo results are combined with measurements of the $\mathrm{CO}$ vibrational lifetime to yield the homogeneous pure dephasing. The pure dephasing is the Fourier transform of the homogeneous linewidth with the lifetime contribution removed. The mutant $\mathrm{H} 64 \mathrm{~V}$ protein's $\mathrm{CO}$ vibrational pure dephasing rate is $\sim 20 \%$ slower (narrower pure dephasing linewidth) than the native protein at all temperatures, although the only difference between the two proteins is the replacement of the native's polar distal histidine by a non-polar valine. The mutant H93G(N-MeIm) pure dephasing is identical to the native's, despite the severing of the only covalent bond between the heme and the globin. These results provide insights into the mechanisms of the transmission of protein fluctuations to the $\mathrm{CO}$ ligand bound at the active site.
\end{abstract}

Keywords: Dephasing; dynamics; echo; myoglobin; protein; vibration

\section{INTRODUCTION}

This paper presents a study of horseheart whale carbonmonoxy myoglobin (Mb-CO) and a comparison of its dynamics to two myoglobin mutants. Myoglobin (Mb), the oxygen carrier in muscle, is a single polypeptide chain of 153 amino acids and has a mass of $18 \mathrm{kD}$ [1]. The capacity of myoglobin to bind oxygen, carbon monoxide, and

\footnotetext{
*Corresponding author.
} 
other ligands depends on the presence of a non-polypeptide prosthetic group protoheme [Fe(II)protoporphyrin IX]. The only polar amino acids side groups internal to the protein pocket that contains the protoheme are two histidines [1]. The proximal histidine is covalently bound to the Fe forming the fifth coordination site of the heme. The sixth coordinate site of the heme is the active site of the protein where the ligand bonds. The distal histidine is physically near the sixth coordinate site of the heme but not directly covalently bonded to it.

When bound to $\mathrm{Mb}$, the $\mathrm{CO}$ vibrational frequency is substantially red shifted from the gas phase frequency and separated into three distinct bands, which are labeled $\mathrm{A}_{0}, \mathrm{~A}_{1}$, and $\mathrm{A}_{3}$ in order of decreasing frequency. These bands, occurring at 1969, 1945, and $1930 \mathrm{~cm}^{-1}$, reflect distinct conformational substates of the protein [2].

A substantial literature exists that explores the structure and binding kinetics of $\mathrm{CO}$ at the active site of $\mathrm{Mb}$ using a variety of techniques, including X-ray crystallograpy [3,4], ${ }^{13} \mathrm{C}$ NMR [5], time dependent optical spectroscopy [6-9], Raman spectroscopy [10, 11], and mid-infrared absorption spectroscopy [12-14]. While these methods yield a wealth of information about the equilibrium structure of $\mathrm{Mb}-\mathrm{CO}$ and the $\mathrm{CO}$ binding kinetics, they provide only indirect dynamical information about the protein with a ligand bound to the active site. Molecular dynamics simulations show that $\mathrm{Mb}$ has a flexible structure in constant motion at room temperature [15]. Such motions can be either on a relatively small scale involving a few of the constituent atoms, such as the torsion of an amino acid residue, or they can be large scale motions involving entire regions of the protein backbone. Simulations over a period of $300 \mathrm{ps}$ indicate that $\mathrm{Mb}$ samples thousands of local energy minima of approximately equal energies, separated by barriers of varying height [15]. These minima correspond to different conformational substates of the protein. It has been proposed that this characteristic of proteins is analogous to the energy landscape in glasses [16].

Recently, the first picosecond infrared vibrational echo experiments on a biological system, myoglobin-CO, were reported [17]. Vibrational echo studies were performed on the $\mathrm{CO}$ stretch $\mathrm{A}_{1}$ line. Pure dephasing is the rate of coherence loss of an ensemble of vibrational transitions with contributions from inhomogeneous broadening and population relaxation $\left(T_{1}\right)$ removed. The rate of pure dephasing, $1 / T_{2}^{*}$ was 
measured from 60 to $300 \mathrm{~K}$. The temperature dependent pure dephasing rate has a functional form that can be described as a power law and an exponentially activated process, [18]

$$
\frac{1}{T_{2}^{*}}=a_{1} T^{\alpha}+a_{2} \mathrm{e}^{-\Delta E / k T}
$$

where $T_{2}^{*}$ is the pure dephasing time, $T$ is temperature, $\alpha$ is the power law exponent, $\Delta E$ is the activation energy and $k$ is Boltzmann's constant. The vibrational echo studies provided insights into how protein dynamics couple to the ligand bound at the protein's active site.

To more fully understand the nature of protein dynamics communicated to the active site in $\mathrm{Mb}$, we also performed a detailed temperature dependent vibrational echo study of a mutant of $\mathrm{Mb}$ in which the polar distal histidine is replaced with non-polar valine $(\mathrm{H} 64 \mathrm{~V})$. Changes in the temperature dependent pure dephasing of the mutant provide insights into the nature of protein dynamics. In this paper, the vibrational echo studies of the $\mathrm{CO}$ dephasing of $\mathrm{H} 64 \mathrm{~V}$ are compared to the previous studies on $\mathrm{Mb}-\mathrm{CO}$ [17]. The results show that replacing the distal histidine with valine is a sufficient modification of the protein to change the $\mathrm{CO}$ vibrational dephasing over the entire temperature range. The vibrational dephasing rate of the mutant is smaller than that of $\mathrm{Mb}$ but has the identical temperature dependence within experimental error. The current experiments are discussed in terms of the model proposed in the context of the $\mathrm{Mb}$ results [17]. In addition, preliminary results on another mutant, H93G(N-MeIm), are also discussed.

\section{THE VIBRATIONAL ECHO METHOD AND EXPERIMENTAL PROCEDURES}

Vibrational line shapes in condensed phases contain the details of the dynamic interactions of a normal mode with its environment [19-21]. However, the line shape can also include essentially static, structural perturbations associated with the distribution of local configurations of the environment of the vibrational oscillator, i.e., inhomogeneous broadening. The IR absorption or Raman line shape represents a 
convolution of the various dynamic and static contributions [22]. The ps infrared vibrational echo experiment is a time domain, nonlinear method that can extract the homogeneous vibrational line shape from an inhomogeneously broadened line [23, 24]. By combining vibrational echo measurements with pump-probe vibrational energy relaxation measurements, the dynamical contributions to a vibrational transition can be elucidated [23].

The echo technique was originally developed as the spin echo in magnetic resonance in 1950 [25]. In 1964, the method was extended to the visible optical regime as the photon echo [26,27]. Since then, photon echoes have been used extensively to study electronic excited state dynamics in many condensed matter systems. Previous optical coherence experiments performed on proteins examined the dephasing of electronic transitions [28]. Because of the rapid dephasing (broad homogeneous linewidths) of electronic transitions, these experiments are only performed at very low temperatures, $<20 \mathrm{~K}$. The vibrational echo experiments enable the use of optical coherence methods to study protein dynamics at physiologically relevant temperatures [17]. Further, vibrational echoes probe dynamics on the ground state potential surface only. Recently, vibrational echoes have been used to examine vibrational dynamics in liquids, glasses [23, 24], and proteins [17].

The vibrational echo experiment uses a two pulse excitation sequence. The first pulse excites a coherent superposition of the $\nu=0$ and $\nu=1$ vibrational levels. Immediately after the first pulse, the vibrational dipoles oscillate in phase. Because there is an inhomogeneous distribution of vibrational transition frequencies, the individual dipoles oscillate with some distribution of frequencies. Thus, the initial phase relationship is rapidly lost. This effect is the vibrational free induction decay. After a time $\tau$, a second pulse, traveling along a path making an angle $\theta$ with that of the first pulse, excites the sample. This second pulse changes the phase factors of each vibrational superposition state in a manner that initiates a rephasing process. At time $2 \tau$, the sample emits a third coherent pulse of light. The emitted pulse propagates along a path that makes an angle $2 \theta$ with the path of the first pulse. The third pulse is the vibrational echo. It is generated when the ensemble of microscopic dipoles is rephased at time $2 \tau$.

The rephasing at $2 \tau$ has removed the effects of the inhomogeneous broadening. However, fluctuations of the environment that are 
coupled to the $\mathrm{CO}$ vibration (homogeneous broadening) cause the frequencies to fluctuate. Thus, at $2 \tau$, the rephasing is not perfect. As $\tau$ is increased, the fluctuations produce larger accumulated phase errors among the microscopic dipoles, and the intensity of the echo is reduced. A measurement of the echo intensity versus $\tau$ is called an echo decay curve. The vibrational echo decay signal, $S(\tau)$, is given by

$$
S(\tau)=S_{0} \mathrm{e}^{-4 \tau / T_{2}}
$$

where $T_{2}$ is the homogeneous dephasing time. The Fourier transform of the echo decay is directly related to the homogeneous line shape [29]. An exponential vibrational echo decay corresponds to a Lorentzian line shape with a width, $\Gamma$, given by

$$
\Gamma=\frac{1}{\pi T_{2}}=\frac{1}{\pi T_{2}^{*}}+\frac{1}{2 \pi T_{1}} .
$$

$T_{2}$ is the homogeneous dephasing time determined from the echo decay constant. $T_{1}$ is the vibrational lifetime determined from the pumpprobe experiments. Measurements of $T_{2}$ and $T_{1}$ permit the determination of $T_{2}^{*}$, the pure dephasing contribution to the linewidth.

The vibrational echo and pump-probe experiments were performed at the Stanford Free Electron Laser (FEL) Center. The FEL pulse train consists of a macropulse having a duration of $\sim 3 \mathrm{~ms}$ and repeating at $10 \mathrm{~Hz}$. Within each macropulse is a series of micropulses repeating at $11.8 \mathrm{MHz}$. Each nearly transform limited Gaussian micropulse had an energy of $\sim 1 \mu \mathrm{J}$. The pulse duration was $\sim 1.7$ ps for the experiments. The FEL frequency, tuned to $1969 \mathrm{~cm}^{-1}$ for the experiments, is actively stabilized to within $0.02 \%$ of the center frequency. Both the autocorrelation and the spectrum were monitored continuously during experiments.

\section{RESULTS AND DISCUSSION}

The temperature dependence of the vibrational echo decays as well as the pump-probe lifetime measurements were obtained from 60 to $300 \mathrm{~K}$ for native $\mathrm{Mb}-\mathrm{CO}$ in $95: 5$ glycerol : water. Each decay was fit to 
a single exponential. Care was taken in each case to verify the stability of the fit, and for most temperatures, the decay constant could be determined within $3 \%$ for the echo data, and $5 \%$ for the pump-probe data. At temperatures below $60 \mathrm{~K}$, the homogeneous dephasing rate is dominated by the lifetime to such an extent that it is not possible to obtain the pure dephasing times accurately.

Figure 1 shows an example of vibrational echo data taken on $\mathrm{MbCO}$ at $60 \mathrm{~K}$ with an exponential fit. As can be seen, the signal to

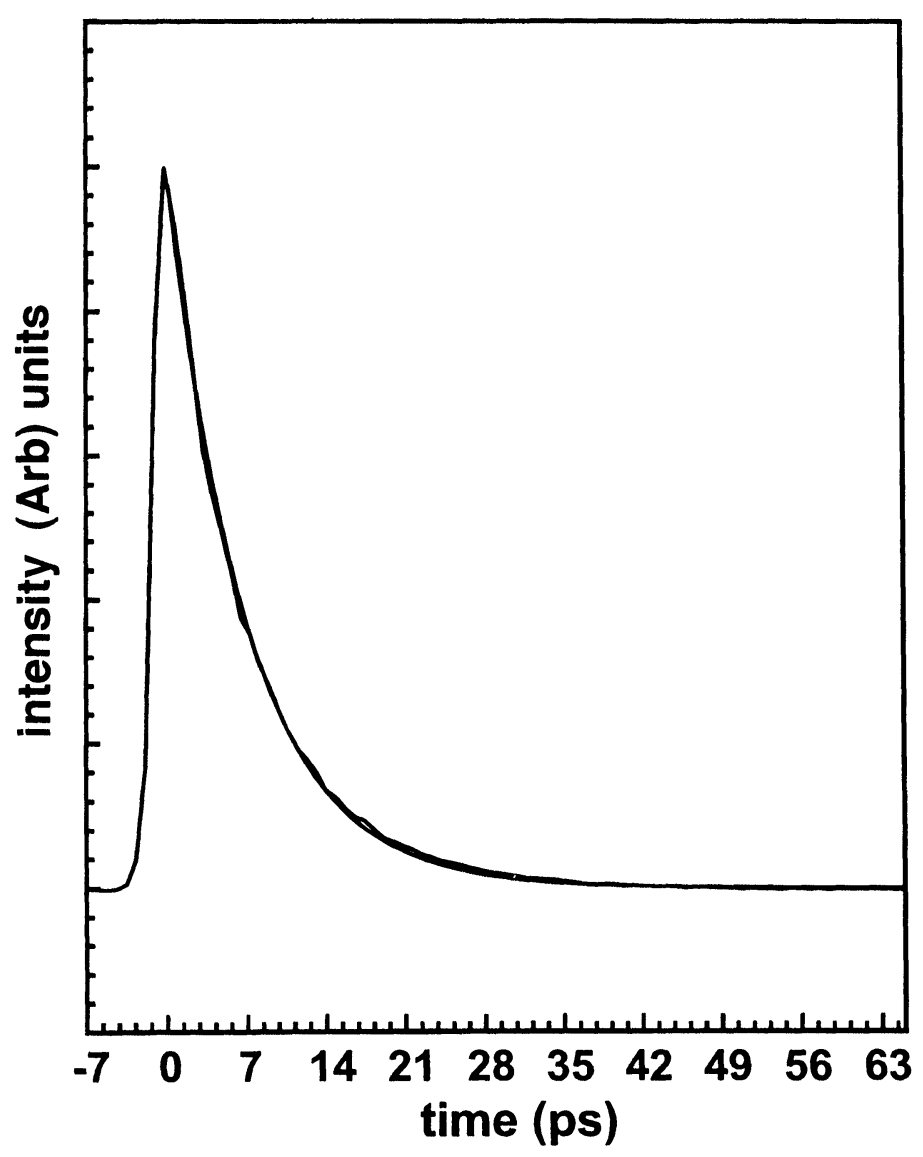

FIGURE 1 Example of vibrational echo data taken on $\mathrm{MbCO}$ at $60 \mathrm{~K}$ and a fit to a exponential. Data of this quality were taken at all temperatures. 
noise is excellent despite the sample's small peak and large background. Data of this quality was taken for all points.

Figure 2 shows temperature dependent data. The triangles are the measured values of $T_{2}$ obtained from the vibrational echo decays using Eq. (2). The squares are $2 T_{1}, T_{1}$ is the decay constant measured in the pump-probe vibrational lifetime experiment. From Eq. (3), it is seen that the relevant quantity is twice the lifetime, or $2 T_{1}$. The circles are $T_{2}^{*}$, the pure dephasing time, obtained from $T_{2}$ and $2 T_{1}$, using Eq. (3). $T_{1}$ has a mild temperature dependence. $T_{2}$ has a steeper temperature

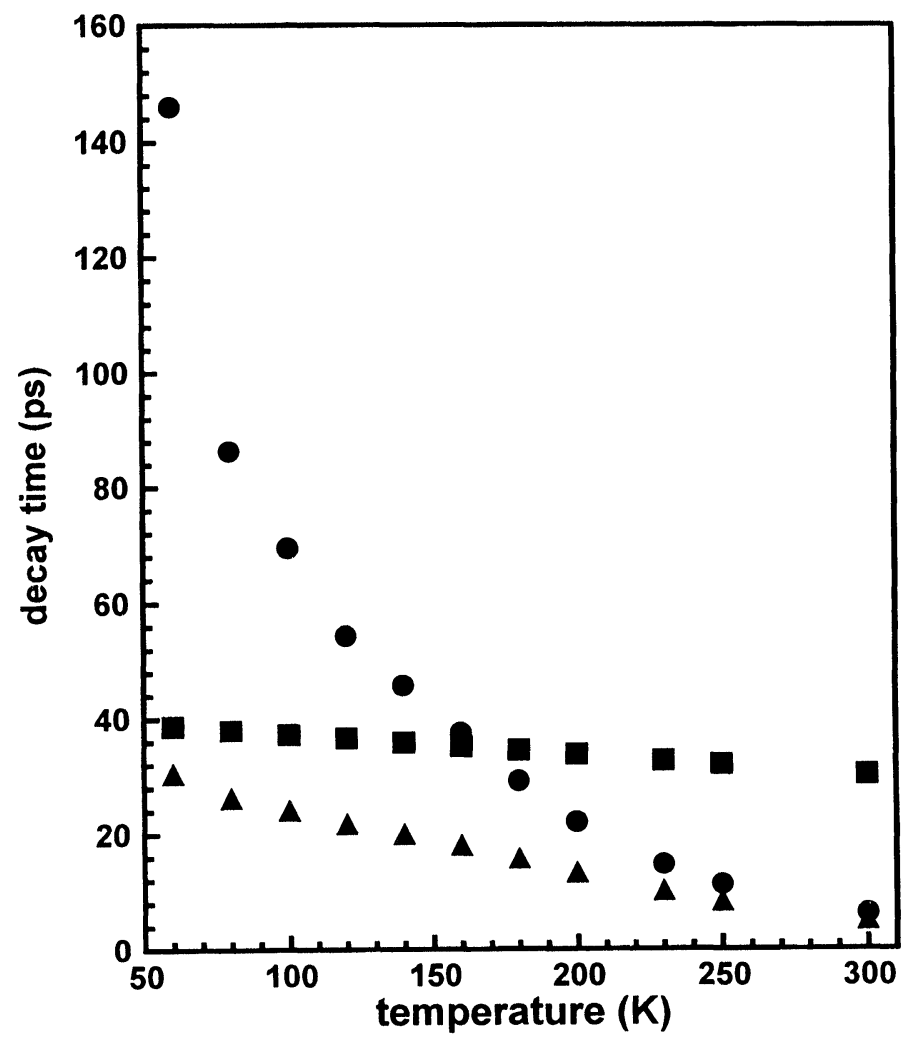

FIGURE 2 Temperature dependent data for native $\mathrm{Mb}$-CO. The triangles are the measured values of $T_{2}$ obtained from the vibrational echo decays using Eq. (2). The squares are $2 T_{1} . T_{1}$ is the decay constant measured in the pump-probe vibrational lifetime experiments. The circles are $T_{2}^{*}$, the pure dephasing time, obtained from $T_{2}$ and $2 T_{1}$, using Eq. (3). 
dependence, changing by a factor of about 8 between 60 and $300 \mathrm{~K}$. The pure dephasing, $T_{2}^{*}$, which arises from the vibrational energy level fluctuations, has a steep temperature dependence. At the lowest temperatures, the lifetime is the major contributor to the homogeneous dephasing (inverse of the linewidth). By room temperature, the lifetime contribution to the homogeneous line is small; the pure dephasing completely dominates the homogeneous dephasing. At all temperatures, the line is inhomogeneously broadened. At room temperature, the homogeneous linewidth is $1.9 \mathrm{~cm}^{-1}$ while the absorption spectrum FWHM width is $\sim 12 \mathrm{~cm}^{-1}$, i.e., the absorption spectrum is about 6 times wider than the homogeneous linewidth.

Figure 3 displays the native pure dephasing rate, $1 / T_{2}^{*}$, on a $\log$ plot. The temperature dependence of the pure dephasing rate is much

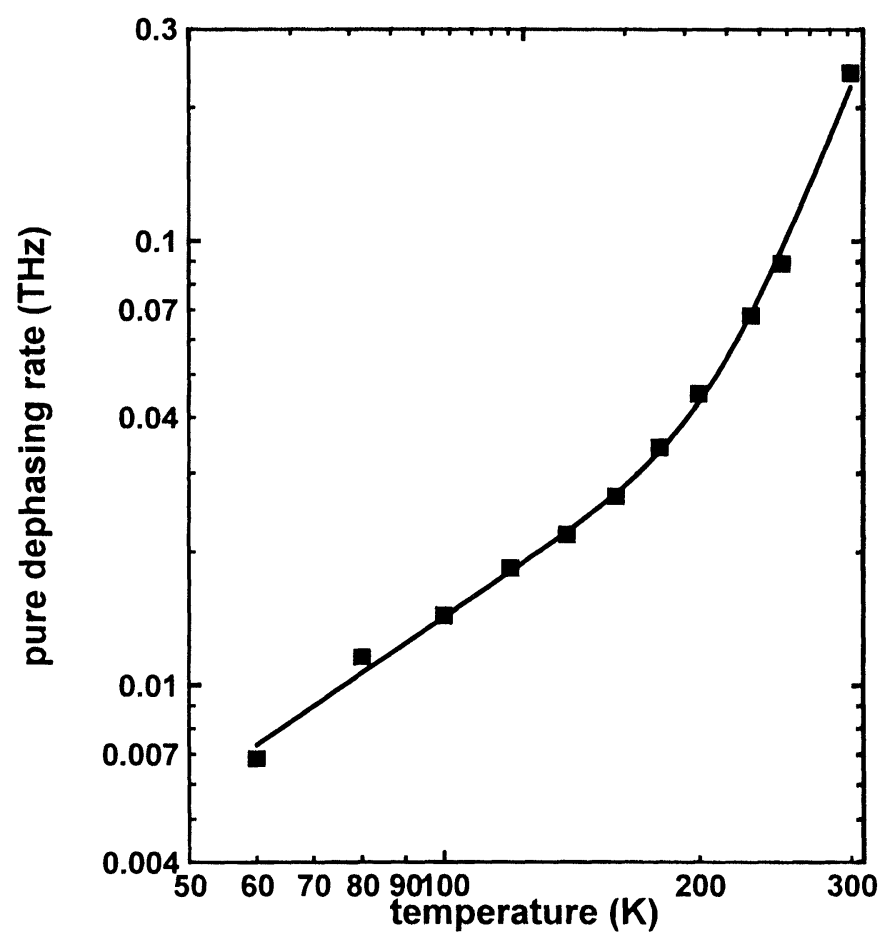

FIGURE 3 Log plot of the pure dephasing rate, $1 / T_{2}^{*}$, versus temperature for native $\mathrm{MbCO}$. Below $\sim 185 \mathrm{~K}$, the data follow a power law, $T^{1.3}$, dependence, which appears linear on the log plot. Above $\sim 185 \mathrm{~K}$, an exponentially activated process describes the data with $\Delta E=\sim 1000 \mathrm{~cm}^{-1}$. 
milder at low temperatures, with a break in the dependence at $\sim 185 \mathrm{~K}$. The data fit well to Eq. (1) [18] ${ }^{1}$. The low temperature data fall on a straight line, suggesting that the temperature dependence can be described by a power law, $T^{\alpha}$. The exponent of the power law is $\alpha=1.3 \pm 0.1$ for all proteins and solvents studied.

The $T^{1.3}$ temperature dependence may arise from tunneling dynamics of a system of protein two level systems (PTLS) [17]. The PTLS are akin to the two level systems of very low temperature glasses. The same statistical mechanics machinery used to describe the low temperature $(\sim 1 \mathrm{~K})$ heat capacities of glasses [30,31] and the optical dephasing of electronic transitions of chromophores in low temperature glasses [32] can be used to describe the PTLS induced vibrational dephasing of $\mathrm{Mb}-\mathrm{CO}$ at much higher temperatures $(\sim 100 \mathrm{~K})$. It needs to be stressed that other physical processes can yield similar temperature dependencies. For example, a power law temperature dependence can arise from activation over barriers rather than tunneling if there is the appropriate broad distribution of activation energies. In either case, the results indicate the existence of a complex protein energy landscape.

It is important to note that the $\sim 200 \mathrm{~K}$ break (coincidentally) occurs at approximately the glass transition temperature of the solvent. However, vibrational echo studies in other solvents, (ethylene glycol, trehalose), also show this break near $\sim 200 \mathrm{~K}$, despite significantly different solvent $T_{g} s$ [33]. These results suggest that there is a fundamental change, perhaps a protein "glass transition", in myoglobin at $\sim 200 \mathrm{~K}$ [18].

In addition to the change in temperature, the reduction in the solvent viscosity with increasing temperature plays an important role in the temperature dependence of the $\mathrm{CO}$ vibrational dephasing at high temperature. The viscosity dependence at fixed temperature will be presented elsewhere [18].

In previous papers on native $\mathrm{Mb}-\mathrm{CO}$ [17], we argued that the motions of the solvent, glycerol/water, were not responsible for the dephasing. For the solvent to cause dephasing, its motions must

\footnotetext{
${ }^{1}$ Eq. (1) fit the dynamics for Mb-CO in a solid sample only. For liquids, the dynamics are affected by changes in sample viscosity as well as changes in temperature. See Rector, K. D., Engholm, J. R., Rella, C. W., Hill, J. R., Dlott, D. D. and Fayer, M. D., J. Chem. Phys., submitted (1998).
} 
couple to the transition frequency of the $\mathrm{CO}$. When molecules go from the gas phase to a condensed phase, there is a shift of electronic and vibrational transition frequencies. This effect is referred to as the solvent shift. Molecular interactions with the condensed matter environment are responsible for line broadening as well as the solvent shift. These phenomena are closely related. The line broadening can be static, giving rise to an inhomogeneous line, or dynamic, giving rise to a homogeneous line. In either case, it is the variations in the solvent shift that cause line broadening.

In $\mathrm{Mb}-\mathrm{CO}$, the nature of the solvent itself has little effect on the $\mathrm{CO}$ vibrational transition frequency. The $\mathrm{Mb}-\mathrm{CO}$ transition frequency is virtually identical in a wide variety of liquids, glasses, crystals, or polymers $[12,34,35]$. The solvent shift is unaffected by the medium surrounding the protein even when the change is from a liquid solvent such as water or a mixture of glycerol/water to a protein crystal [35]. In contrast, the frequency difference between the $\mathrm{A}_{0}$ and $\mathrm{A}_{1}$ lines in $\mathrm{Mb}$ $\mathrm{CO}$ is $24 \mathrm{~cm}^{-1}$ [12]. This difference is caused by a change in conformation of the protein, especially the distal histidine. Changes in the protein structure can have a major influence on the $\mathrm{CO}$ vibrational frequency while changes in the solvent have a negligible influence. This spectroscopic information leads to the reasonable conclusion that fluctuations of the protein structure will cause homogeneous dephasing while fluctuations of the solvent structure will not. The solvent does provide a heat bath and a boundary condition that are intimately involved in the protein fluctuations and the dephasing, but the dephasing does not arise from direct coupling of the solvent dynamics to the $\mathrm{CO}$ transition frequency.

In discussing the dephasing experiments on $\mathrm{Mb}-\mathrm{CO}$ [17], it was proposed that fluctuating electric fields produced by time dependent protein structural fluctuations are responsible for $\mathrm{CO}$ vibrational dephasing. It is well established from work on Mbs and other metal carbonyls [36 - 39] that changes in the back of donation of electron density (back bonding) into the $\mathrm{CO} \pi^{*}$ anti-bonding molecular orbital are responsible for static shifts of the $\mathrm{CO}$ vibrational frequency. Increased back bonding weakens the $\mathrm{CO}$ bond and red shifts the vibrational frequency. In porphyrins, the metal $d_{\pi}$, nitrogen $p_{\pi}$, and carbon $p_{\pi}$ electrons form a $\pi$ electron system that is delocalized across the heme (or heme-like) macrocycle. It is this delocalized $d_{\pi} / p_{\pi}$ system that back 
donates electron density to the $\mathrm{CO} \pi^{*}$ molecular orbital. Experiments by Oldfield et al. [5] indicate that in different Mb's, variations in electric fields resulting from different protein conformations are responsible for changes in back bonding, and, therefore, the observed static shifts in vibrational frequency $[39,40]$.

In the proposed fluctuating electric field dephasing mechanism, the dynamics of the amino acids, particularly the ones with polar side groups, produce fluctuating electric fields near the heme macrocycle. The fluctuating fields generate time dependent variations in the macrocycle's electron density distribution, which, in turn, cause time evolution of the magnitude of the back bonding. It is this time dependent back bonding that is responsible for the $\mathrm{CO}$ vibrational energy fluctuations, i.e., the vibrational pure dephasing.

In the protein pocket that contains the heme- $\mathrm{CO}$, there are two highly polar amino acids, the proximal histidine and the distal histidine. The distal histidine is not directly bound to the heme-CO.

Figure 4 compares the data for the mutant $\mathrm{H} 64 \mathrm{~V}-\mathrm{CO}$ (diamonds) and $\mathrm{Mb}-\mathrm{CO}$ (squares). The dephasing rate for $\mathrm{H} 64 \mathrm{~V}-\mathrm{CO}$ is slower than for $\mathrm{Mb}$ over the entire temperature range. Comparing the data points at each temperature reveals that the H64V-CO data is $21 \% \pm 3 \%$ slower than the $\mathrm{Mb}$ data with no systematic variation. A solid line through the native $\mathrm{Mb}-\mathrm{CO}$ data was obtained from a fit of Eq. (3) to the data. This line was translated down with no change in shape or scale, and is the line through the H64V data. The translation on the $\log$ plot is the same as multiplying the function that passes through the $\mathrm{Mb}-\mathrm{CO}$ by a constant. It can be seen that within experimental error, the functional form of the two data sets is identical.

This decrease in the pure dephasing rate is consistent with and provides support for the fluctuating electric field induced dephasing mechanism. The structure of the pocket differs only at a single residue. However, this difference is significant. The interaction between $\mathrm{CO}$ and the polar distal histidine is replaced by a weaker interaction between $\mathrm{CO}$ and the nonpolar valine. X-ray crystallographic data of this mutant show the equilibrium structure of the protein is not significantly different from that of myoglobin [4]. Therefore, it is unlikely that there is a significant change in the global dynamics of the protein. Thus, the temperature dependence of the $\mathrm{CO}$ vibrational pure dephasing is unchanged since it reflects the spectrum of protein 


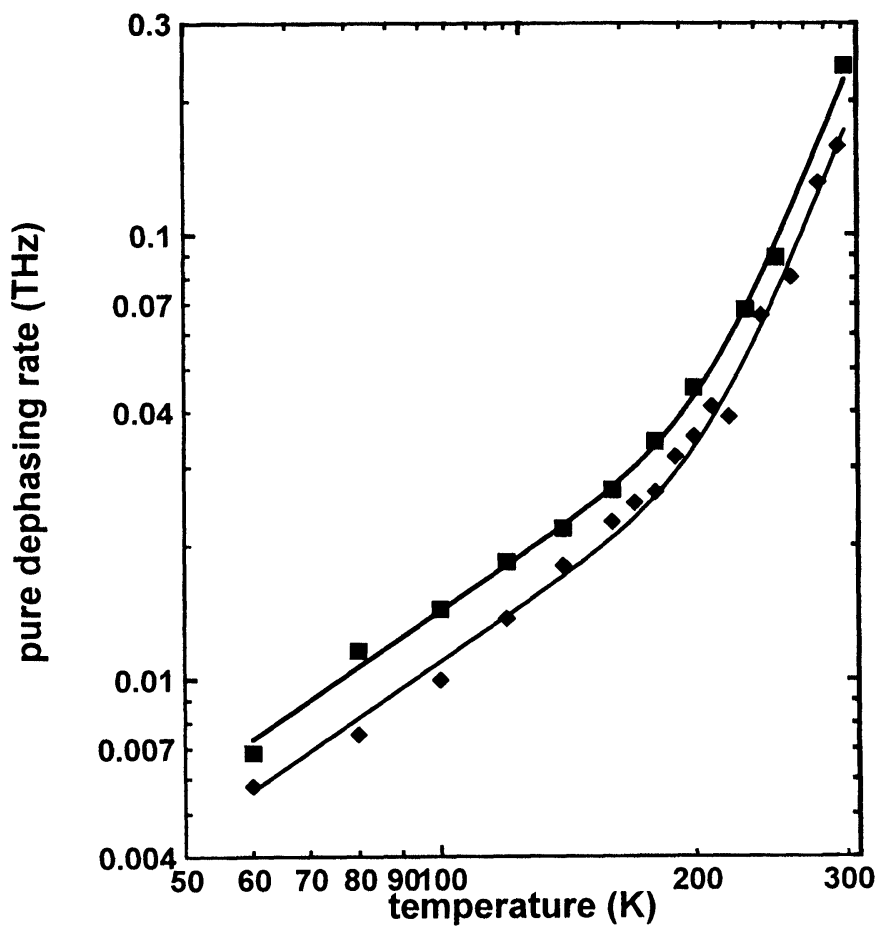

FIGURE 4 Log plot of the pure dephasing rate, $1 / T_{2}^{*}$, versus temperature for H64V-CO (diamonds) and $\mathrm{Mb}-\mathrm{CO}$ (squares). The solid line through the H64V-CO data was obtained by translating the fit of the $\mathrm{Mb}-\mathrm{CO}$ data down. No other change in the line was made. A vertical translation on a $\log$ plot is the equivalent of multiplying a function by a constant. This demonstrates that the two data sets have the identical functional form of the temperature dependence within experimental error.

fluctuations that are coupled to the CO. However, the strength of the coupling of the protein fluctuations to the $\mathrm{CO}$ will be reduced because one of the closest sources of the fluctuating electric field has been removed.

The reduced coupling of the protein to the heme is also evidenced by the change in the $\mathrm{CO}$ vibrational frequency in $\mathrm{H} 64 \mathrm{~V}$ compared to the dominant $\mathrm{A}_{1}$ line of $\mathrm{Mb}$. The $\mathrm{A}_{1}$ line of $\mathrm{Mb}$ is at $1945 \mathrm{~cm}^{-1}$ while the $\mathrm{H} 64 \mathrm{~V}$ line is at $1969 \mathrm{~cm}^{-1}$. The higher frequency is caused by less back bonding. In previous studies of the vibrational lifetimes of Mbs and model heme compounds, it was found that there was a direct correlation between the $\mathrm{CO}$ absorption frequency and the lifetime [41 -44]. 
Higher vibrational transition frequency was linearly associated with a longer vibrational lifetime. This demonstrated that the vibrational lifetime was determined by coupling to the heme $\pi$ electron system via the back bonding interaction. Increased back bonding caused by stronger electric field interactions with the protein results in a shift of the absorption to lower energy and a shorter vibrational lifetime. In the current experiments, the $\mathrm{CO}$ stretch frequency of H64V-CO absorbs at higher frequency than the $\mathrm{Mb} \mathrm{A}_{1}$ line because of a decrease in the electric field induced back bonding. The reduction in static coupling is associated with weaker coupling of the protein fluctuations to the heme macrocycle and thus to the slower dephasing in H64V-CO observed in these vibrational echo experiments.

In the absence of the distal histidine in $\mathrm{H} 64 \mathrm{~V}-\mathrm{CO}$, pure dephasing can still occur because of the motions of the proximal histidine and fluctuations of the more distant parts of the protein. One might presume that the proximal histidine plays a unique role in the dephasing because it is the only covalent link between the heme and the protein. Motions of the $\mathrm{Fe}$ and the protein are coupled through the proximal histidine [1]. Therefore, in addition to an electric field effect, the proximal histidine motions may produce a "mechanical" contribution to the pure dephasing [17].

Preliminary experimental results on another mutant, H93G (NMeIm) provided insights into this model. H93G is a mutant of myoglobin with the proximal histidine replace with a glycine. Then, exogenous $N$-methylimidazole is bound at the $\mathrm{Fe}$ and fills the proximal cavity. The net result is a system with remarkably similar electronic environment to native $\mathrm{Mb}-\mathrm{CO}$ yet the single covalent bond between the herne and the globin is severed [45-46]. The results show that the mutant $\mathrm{H} 93 \mathrm{G}(\mathrm{N}-\mathrm{MeIm})$ temperature dependent pure dephasing is identical within experimental error to the native $\mathrm{Mb}$. [Data not shown] [48]. These results indicate that the mechanical mechanism is not significant in myoglobin.

\section{CONCLUDING REMARKS}

The vibrational echo experiments provide a method for the removal of the inhomogeneous broadening from a vibrational spectrum yielding 
the dynamical information contained in the homogeneous spectrum. We have performed vibrational echo and lifetime relaxation measurements on $\mathrm{CO}$ bound to the active site of myoglobin and two myoglobin mutants. Combining the results of the echo with pumpprobe data, we have obtained the pure dephasing times, $T_{2}^{*}$, at a series of temperatures from 60 to $300 \mathrm{~K} . T_{2}^{*}$ is a measure of the vibrational energy level fluctuations induced by conformational fluctuations of the protein.

The general mechanism proposed previously [17] to explain the coupling of conformational fluctuations of the protein to the vibrational transition energy to cause pure dephasing is suppcrted by this work. The model stated that protein motions produce fluctuating electric fields which give rise to the time dependent back bonding. Replacing the polar distal histidine with the non-polar valine removes one source of the fluctuating electric fields, thus reducing the coupling between the protein fluctuations and the measured pure dephasing. The observation that the rate of pure dephasing observed in $\mathrm{H} 64 \mathrm{~V}-\mathrm{CO}$ is reduced significantly compared to $\mathrm{Mb}-\mathrm{CO}$ is consistent with the model. The picture that emerges is that the heme acts as an antenna that receives and communicates protein fluctuations to the vibration of the $\mathrm{CO}$ ligand bound at the active site. The observation that the rate of pure dephasing of $\mathrm{H} 93 \mathrm{G}(\mathrm{N}-\mathrm{MeIm})$ is identical to native $\mathrm{Mb}-\mathrm{CO}$ verified that the proximal histidine mechanical effects on $\mathrm{CO}$ dephasing is not significant.

\section{Acknowledgments}

The authors acknowledge Dr. C. W. Rella, Dr. J. R. Hill, Prof. D. D. Dlott for significant contributions in obtaining these data and interpretation of these results. Also, the authors thank Prof. S. G. Sligar [H64V] and Prof. S. Boxer [H93G(N-MeIm)] for donation of the mutant proteins. Further, the authors thank Prof. Alan Schwettman and Prof. Todd Smith and their research groups for running the Stanford FEL during these experiment. This research was supported by the Medical Free Electron Laser Program, through the Office of Naval Research, (N00014-94-1-1024). Additional support was provided by the National Science Foundation, Division of Materials Research (DMR93-22504). 


\section{References}

[1] Stryer, L. (1988). Biochemistry, 3rd Ed. (W. H. Freeman and Co., New York).

[2] Austin, R. H., Beeson, K., Eisenstein, L., Frauenfelder, H., Gunsalus, I. C. and Marshal, V. P. (1974). Phys. Rev. Lett., 32, 403.

[3] Kuriyan, J. W., Karplus, M. and Petsko, G. A. (1986). J. Mol. Biol., 192, 133.

[4] Quillin, M. L., Arduini, R. M., Olson, J. S. and Phillips, G. N. Jr. (1993). J. Mol. Biol., 234, 140.

[5] Oldfield, E., Guo, K., Augspurger, J. D. and Dykstra, C. E. (1991). J. Am. Chem. Soc., 113, 7537.

[6] DeBrunner, P. G. and Franuenfelder, H. (1982). Ann. Rev. Phys. Chem., 33, 283.

[7] Frauenfelder, H., Parak, F. and Young, R. D. (1988). Ann. Rev. Biophys. Chem., $17,471$.

[8] Owrutsky, J. C., Li, M., Locke, B. and Hochstrasser, R. M. (1995). J. Phys. Chem., 99, 4842.

[9] Jackson, T. A., Lim, M. and Anfinrud, P. A. (1994). Chemical Physics, 180, 131.

[10] Petrich, J. W. and Martin, J. L. (1989). In: Time-Resolved Spectroscopy, Edited by Clark, R. J. H. and Hester, R. E. (Wiley, New York) p. 335.

[11] Friedman, J. M., Rousseau, D. L. and Ondrias, M. R. (1982). Ann. Rev. Phys. Chem., 33, 471.

[12] Ansari, A., Beredzen, J., Braunstein, D., Cowen, B. R., Frauenfelder, H., Hong, M. K., Iben, I. E. T., Johnson, J. B., Ormos, P., Sauke, T., Schroll, R., Schulte, A., Steinback, P. J., Vittitow, J. and Young, R. D. (1987). Biophys. Chem., $26,337$.

[13] Alben, J. O. and Caughy, W. S. (1968). Biochem., 7, 175.

[14] Alben, J. O., Beece, D., Browne, S. F., Doster, W., Eisenstein, L., Frauenfelder, H., Good, D., McDonald, J. D., Marden, M. C., Moh, P. P., Reinisch, L., Reynolds, A. H., Shyamsunder, E. and Yue, K. T. (1982). Proc. Natl. Acad. Sci. USA, 79, 3744.

[15] Elber, R. and Karplus, M. (1987). Science, 235, 318.

[16] Iben, I. E. T., Basunstein, D., Doster, W., Frauenfelder, H., Hong, M. K., Johnson, J. B., Luck, S., Ormos, P., Schulte, A., Steinback, P. J., Xie, A. and Young, R. D. (1989). Phys. Rev. Lett., 62, 1916.

[17] Rella, C. W., Rector, K. D., Kwok, A. S., Hill, J. R., Schwettman, H. A., Dlott, D. D. and Fayer, M. D. (1996). J. Phys. Chem., 100, 15620.

[18] Gordon, R. G. (1965). J. Chem. Phys., 43, 1307.

[19] Gordon, R. G. (1968). Adv. Magn. Reson., 3, 1.

[20] Berne, B. J. (1971). Physical Chemistry: An Advanced Treatise (Academic Press, New York).

[21] Loring, R. F. and Mukamel, S. (1985). J. Chem. Phys., 83, 2116.

[22] Tokmakoff, A., Zimdars, D., Urdahl, R. S., Francis, R. S., Kwok, A. S. and Fayer, M. D. (1995). J. Phys. Chem., 99, 13310.

[23] Tokmakoff, A. and Fayer, M. D. (1995). J. Chem. Phys., 102, 2810.

[24] Hahn, E. L. (1950). Phys. Rev., 80, 580.

[25] Kurnit, N. A., Abella, I. D. and Hartmann, S. R. (1964). Phys. Rev. Lett., 13, 567.

[26] Abella, I. D., Kurnit, N. A. and Hartmann, S. R. (1966). Phys. Rev. Lett., 14, 391.

[27] Leeson, D. T. and Wiersma, D. A. (1995). Phys. Rev. Lett., 74, 2138.

[28] Farrar, T. C. and Becker, D. E. (1971). Pulse and Fourier Transform NMR (Academic Press, New York).

[29] Phillips, W. A. (1981). Amorphous Solids, Low Temperature Properties, Topics in Current Physics (Springer, Berlin).

[30] Stevels, J. M. (1962). In: Thermodynamics of Liquids and Solids, Edited by Flügge, S. (Springer-Verlag, Berlin), p. 13.

[31] Narasimhan, L. R., Littau, K. A., Pack, D. W., Bai, Y. S., Elschner, A. and Fayer, M. D. (1990). Chemical Rev., 90, 439. 
[32] Hill, J. R., Dlott, D. D. and Fayer, M. D. (1996). Unpublished results.

[33] Ivanov, D., Sage, J. T., Keim, M., Powell, J. R., Asher, S. A. and Champion, P. M. (1994). J. Am. Chem. Soc., 116, 4139.

[34] Cotton, F. A. and Wilkinson, G. (1988). Advanced Inorganic Chemistry (Wiley Interscience, New York).

[35] Boldt, N. J., Goodwill, K. E. and Bocian, D. F. (1988). Inorg. Chem., 27, 1188.

[36] Spiro, T. G. (1983). Iron Porphyrins (Addison-Wesley, Reading, MA).

[37] Li, X. Y. and Spiro, T. G. (1988). J. Am. Chem. Soc., 110, 6024.

[38] Park, K. D., Guo, K., Adebodun, F., Chiu, M. L., Sligar, S. G. and Oldfield, E. (1991). Biochemistry, 30, 2333.

[39] Dlott, D. D., Fayer, M. D., Hill, J. R., Rella, C. W., Suslick, K. S. and Ziegler, C. J. (1996). J. Am. Chem. Soc., 118, 7853.

[40] Hill, J. R., Dlott, D. D., Fayer, M. D., Rella, C. W., Rosenblatt, M. M., Suslick, K. S. and Ziegler, C. J. (1996). J. Phys. Chem., 100, 218.

[41] Hill, J. R., Dlott, D. D., Rella, C. W., Peterson, K. A., Decatur, S. M., Boxer, S. G. and Fayer, M. D. (1996). J. Phys. Chem., 100, 12100.

[42] Hill, J. R., Rosenblatt, M. M., Ziegler, C. J., Suslick, K. S., Dlott, D. D., Rella, C. W. and Fayer, M. D. (1996). J. Phys. Chem., 100, 18023.

[43] Barrick, D. (1994). Biochem., 33, 6546.

[44] Decatur, S. M., DePillis, G. D. and Boxer, S. G. (1996). Biochem., 35, 3925.

[45] DePillis, G. D., Decatur, S. M., Barrick, D. and Boxer, S. G. (1994). J. Am. Chem. Soc., 116, 6981.

[46] Rector, K. D., Engholm, J. R., Rella, C. W., Hill, J. R., Hu, R., Boxer, S. G., Dlott, D. D. and Fayer, M. D. (1998). J. Phys. Chem. B, 102(2), 331. 